\title{
Probabilistic Triadic Model: Relation of Triadic Closure and Social Influences
}

\author{
Rahul Saha ${ }^{1}$, G.Geetha ${ }^{2}$ and Gulshan Kumar $^{3 *}$ \\ ${ }^{1,2}$ School of Computer Applications, Lovely Professional University, India \\ ${ }^{3}$ School of Computer Science of Engineering, Lovely Professional University, \\ India \\ rsahaaot@gmail.com ${ }^{1 *}$,gitaskumar@yahoo.com ${ }^{2}$,gulshan_acet@yahoo.com ${ }^{3}$
}

\begin{abstract}
Triadic closure is the smallest unit of social network analysis building blocks. It helps to interpret the underlying nature of network behaviour and dynamics. Triadic closures may or may not depend on the social influences. In this paper, we have shown an extended theory to measure the probabilistic formulation of triadic closure. Our proposed Probabilistic triadic Model depends upon the probability of social influences which was missing in the existing theories. We have introduced a new parameter called index of inclination for the probability of triadic closure formation. Observations are analysed and it shows how our probability relation between triadic closures or the link formation and social influences precisely interprets all the social networking scenarios.
\end{abstract}

Keywords: social networks, triadic closures, social influences, mutual friends, probability

\section{Introduction}

Social networking may be a network wherever technological aspects area unit born the people communicate to alternative person with some psychological illation, interference and influence. The current world of communication has raised its most urge to mull over upon such communication eventualities. Facebook, Twitter, WhatsApp and many more applications and websites has become our integral a part of our life. Such applications and systems have created the emotional attachment between two users frequent, random and straightforward. Billions of users day and night area unit active with such technology making an enormous network that connects with world relationship consequently. But, terribly rare we've thought of the behaviour of the users whereas making such communications on-line and once there are a unit billions of user's addicted to such applications, there's a good resource of information to be analysed to envision out the inner core of the social networking analysis. Therefore, during this analysis proposal we've projected to research the social links primarily based upon sturdy and weak ties among the users and their interrelatedness.

Triadic closure ${ }^{1,2}$ is a basic aspect of social networking analysis. It provides a local view of the network scenario where the nodes represent the social actors. Triadic closures create the basic building block of any social knit groups and their dynamics ${ }^{3,4}$. Therefore the centrality measures depend on the number of triads in a network. More the triads are in a network, the probability of the global friendship existence increases and as a result the network converges to the static aspects where the interplay of social influence and selection of a bond gets neutral. 


\section{Related Work}

The authors in the paper ${ }^{2}$ have investigated the triadic closure formation with the factors of homophily, geographical locations and interactions. In the paper ${ }^{3}$ the authors have shown a static network model where they have established three scaling relations: node distribution, scaling of attachment kernel and the cluster coefficient. The work in the paper $^{4}$ deals with the coevolution of a model. This is dynamic and driven locally with preferential triadic closure. The paper ${ }^{5}$ shows about two metrics link delay and triadic closure delay which significantly attributed for some factors of social network analysis such as network maturity, environmental and social contexts. The study in the paper ${ }^{1}$ shows the effect of assertive mixing, preferential attachment and triadic closures in domain of citation networks. The authors have used exponential random graph approach. The authors in the paper ${ }^{6}$ have proposed a model suitable for dynamic network topologies. They have also shown the bistability ${ }^{6}$ relationship with triadic closures in a dynamic environment. The work in the paper $^{7}$ has shown the driving forces in the collaboration network. The authors have investigated the effect of geographical locations and triadic closures on such networks. In paper ${ }^{8}$, the authors have introduced the directed closure concepts to deal with transitivity aspects of the real networks and also have shown a differential relation between preserving reciprocity and the closures. In paper ${ }^{9}$, the authors have analysed the behaviour of closed triads in dynamic networks.

In all of these work done previously, different analysis have been done on triads but the willingness of the human being for a particular link creation is missing which has been addressed by us in this paper. The rest of the paper is organized as: Section 3 describes the basic baseline model, section 4 describes our proposed probabilistic triadic model, Section 5 analyses our experimentation and observation and finally we conclude in Section 6.

\section{Existing Theory: Baseline Model}

The baseline model as shown in the previous work ${ }^{8,9}$ says that $\mathrm{k}$ is the number of mutual friends who can provide independent probability $\mathrm{p}$ to their corresponding friends whether to accept a link or reject a link. So, if $\mathrm{p}$ is the probability of accepting a link of a friend, then the probability of not forming that link will be (1-p). For k mutual friends, this will become as $(1-p)^{k}$ as number of $\mathrm{k}$ are independent trials. So, for $\mathrm{k}$ mutual friends, the probability of forming a link is given by,

$$
T_{\text {baseline }}(k)=1-(1-p)^{k}
$$

where, $T_{\text {baseline }}(k)$ is the probability of formation $\mathrm{k}$ new links.

The above baseline model is also considered as the basis of depicting the nature of triadic closure formation in a network. Therefore, the above equation can be written in form of the triadic closure as shown below.

$$
T_{\text {triadic }}(k)=1-(1-p)^{k}
$$

where, $\mathrm{P}_{\text {Triadic }}$ is the probability of generating triadic closures.

We all know that social influences help in creating triadic closures but how they mathematically behave, was missing in the literature review as well as in the baseline model.

\section{Proposed Theory: Probabilistic Triadic Model}

Triadic closure depicts the socio-psychological relationships where true friends having a mutual friend also become friend of each other with a temporal span and corresponding to justified social influence or foci affluence. Here, we have considered two factors 
simultaneously: social effects and time span. Each of these factors has their own probability to occur in a social network. Let us assume the probability factor of social influence is $\mathrm{P}_{\text {social }}$. This probability will increase as the time span increases. We can formulate this as:

$$
P_{\text {social }} \propto T \text { where } \mathrm{T} \text { denotes time. }
$$

Now, $\mathrm{P}_{\text {social }}=0$, if $\mathrm{T}=0$ and $\mathrm{P}_{\text {social }} \rightarrow 1$ if $\mathrm{T}$ increases eventually

In general, the measurement for $\mathrm{P}_{\text {social }}$ can be given as:

$$
P_{\text {social }}=\varrho^{k}
$$

where $\mathrm{k}$ is the number of mutual friends in a particular context that creates individual probability of influence and $\varrho$ must be $>0.5$ to have the effect of influence.

Considering the existing theory of measuring triadic closure we can say that, the independent probability $\mathrm{p}$ that $\mathrm{k}$ mutual friends provide, is ultimately the sum of probability of all the social influences over time $\mathrm{T}$. If $\mathrm{k}$ is the number of mutual friends it means $\mathrm{k}$ conditions of social influences will exist. We have introduced a term "index of inclination" for individual user and or user groups to a particular context of relation. The definition of this parameter is given as:

Index of Inclination is a parametric value to identify an individual's own interest to incline for a particular context of communication. The value for this index ranges from 0 to 1 . The higher the value is the more inclination to a particular context has been signified. This value will help to generalize the effect of triadic closures and homophily and will provide a realistic analysis of the social networking observations.

Now, to generalize the concept with individual's inclination, the overall probability of social influences become as:

$$
P_{\text {social }}=\varrho^{k} \times p_{\text {inc }}
$$

Where, $p_{\text {inc }}$ is the index of inclination of a user to a particular context and it must be $>0.5$ for a particular context from which the mutual friends belong too.

Therefore, the overall triadic closure probability can be given as:

$$
P_{\text {triadic }}=1-(1-p)^{\varrho^{k} \times p_{\text {inc }}}
$$

One interesting behaviour that we can notice in this probability model is that if the value of $p_{i n c}$ increase, the probability of the triadic closures $P_{\text {triadic }}$ also increases irrespective of the value of $\varrho^{k}$.

From the model above, we can draw the theorem of probabilistic triadic model as below.

Theorem 1: Probabilistic Triadic Model is a mathematical model that depends on the value of inclination index $p_{\text {inc }}$, where $p_{\text {inc }}>0.5$.

Theorem 2: Probabilistic Triadic Model works with social influences in conjunction with the number of mutual friends.

The proofs of the above two theorems have been analysed through the experimental results and observation data. 


\section{Experimental Observation and discussion}

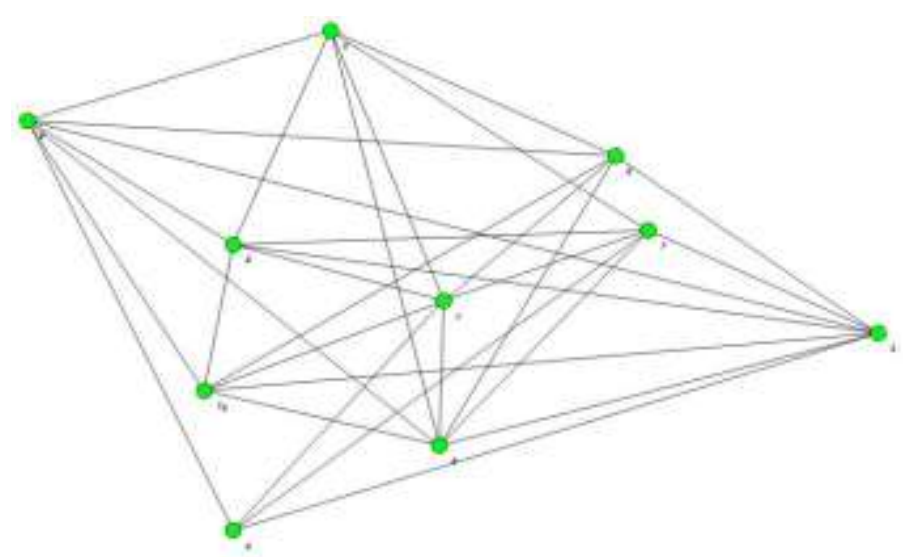

Figure 1. Connection Graph of Observation in Table-1

We have observed 50 random Facebook users but due to the limitation of data size publication we have shown here, 10 random Facebook users with mutual friends. Observation was done in the last one month from May 20, 2016 to August 20, 2016. The users and their corresponding mutual friends are attached with the contexts that have been classified in two categories: common organization [Context 1] and travel hobby [Context 2]. We can notify the relationship in the following relationship matrix where the notation " $\mathrm{b} / \mathrm{n}$ " signifies as if $\mathrm{b}$ is 1 it means two users are friends and if it 0 it means no relation between the users and value of $n$ is either 1 or 2 depending upon context 1 or context 2 . Names of the users are withdrawn for the confidentiality of the identity. Table 1 shows the start point scenario of our observation and the relation matrix is graphically shown in Figure-1. Table 2 shows the observation after three months as stated above.

Table 1. Observation in Month of May, 2016

\begin{tabular}{|c|c|c|c|c|c|c|c|c|c|c|}
\hline & $\mathrm{U} 1$ & $\mathrm{U} 2$ & $\mathrm{U} 3$ & $\mathrm{U} 4$ & $\mathrm{U} 5$ & $\mathrm{U} 6$ & $\mathrm{U} 7$ & $\mathrm{U} 8$ & $\mathrm{U} 9$ & $\mathrm{U} 1$ \\
& & & & & & & & & 0 \\
\hline $\mathrm{U} 1$ & - & $1 / 1$ & 0 & $1 / 1$ & $1 / 1$ & $1 / 1$ & $1 / 1$ & 0 & $1 / 1$ & $1 / 1$ \\
\hline $\mathrm{U} 2$ & $1 / 1$ & - & $1 / 2$ & 0 & $1 / 2$ & $1 / 1$ & 0 & $1 / 1$ & 0 & $1 / 1$ \\
\hline $\mathrm{U} 3$ & 0 & $1 / 2$ & - & $1 / 1$ & 0 & $1 / 2$ & $1 / 1$ & $1 / 1$ & $1 / 1$ & $1 / 1$ \\
\hline $\mathrm{U} 4$ & $1 / 1$ & 0 & $1 / 1$ & - & 0 & 0 & $1 / 1$ & $1 / 1$ & 0 & 0 \\
\hline U5 & $1 / 1$ & $1 / 2$ & 0 & 0 & - & $1 / 2$ & $1 / 1$ & $1 / 1$ & $1 / 1$ & 0 \\
\hline $\mathrm{U} 6$ & $1 / 1$ & $1 / 1$ & $1 / 2$ & 0 & $1 / 2$ & - & $1 / 1$ & $1 / 1$ & 0 & $1 / 2$ \\
\hline $\mathrm{U} 7$ & $1 / 1$ & 0 & $1 / 1$ & $1 / 1$ & $1 / 1$ & $1 / 1$ & - & 0 & $1 / 1$ & 0 \\
\hline $\mathrm{U} 8$ & 0 & $1 / 1$ & $1 / 1$ & $1 / 1$ & $1 / 1$ & $1 / 1$ & 0 & - & $1 / 1$ & $1 / 2$ \\
\hline U9 & $1 / 1$ & 0 & $1 / 1$ & 0 & $1 / 1$ & 0 & $1 / 1$ & $1 / 1$ & - & $1 / 1$ \\
\hline $\begin{array}{c}\mathrm{U} 1 \\
0\end{array}$ & $1 / 1$ & $1 / 1$ & $1 / 1$ & 0 & 0 & $1 / 2$ & 0 & $1 / 2$ & $1 / 1$ & - \\
\hline
\end{tabular}


Table 2. Probable Triadic Closure

\begin{tabular}{|l|l|l|l|l|l|l|l|}
\hline $\begin{array}{l}\text { Probable } \\
\text { link due } \\
\text { to triadic } \\
\text { closure } \\
\text { property } \\
\text { (user no.) }\end{array}$ & $\begin{array}{l}\text { Mutual } \\
\text { Friends } \\
\text { (user no.) }\end{array}$ & $\begin{array}{l}\text { No. } \\
\text { Mutual } \\
\text { friends } \\
\text { with } \\
\text { context } \\
1\end{array}$ & $\begin{array}{l}\text { No. } \\
\text { Mutual } \\
\text { friends } \\
\text { with } \\
\text { context } \\
2\end{array}$ & $\begin{array}{l}\text { No. Mutual } \\
\text { friends } \\
\text { with both } \\
\text { context 1 } \\
\text { and context } \\
2\end{array}$ & $\begin{array}{l}\text { Users' } \\
\text { inclination } \\
\text { index } \\
\text { context 1 } \\
(>5)\end{array}$ & $\begin{array}{l}\text { Users' } \\
\text { inclination } \\
\text { index } \\
\text { context 2 } \\
(>5)\end{array}$ & $\begin{array}{l}P_{\text {triadic }} \\
\text { (with non } \\
\text { zero } \\
\text { inclination } \\
\text { index) }\end{array}$ \\
\hline$\{4,5\}$ & $2,4,6,9,10$ & 3 & 0 & 2 & 0 & 0.8 & 0.130 \\
\hline$\{4,6\}$ & $1,7,8$ & 3 & 0 & 0 & 0.9 & 0 & 0.075 \\
\hline$\{1,8\}$ & $2,2,7,8$ & 3 & 0 & 1 & 0.9 & 0 & 0.038 \\
\hline$\{2,4\}$ & $1,3,8$ & 2 & 0 & 1 & 0.7 & 0.8 & 0.242 \\
\hline$\{2,7\}$ & $1,3,5,6$, & 2 & 0 & 2 & 0 & 0.9 & 0.059 \\
\hline$\{2,9\}$ & $1,3,5,8,10$ & 3 & 0 & 2 & 0.7 & 0 & 0.144 \\
\hline$\{3,5\}$ & $2,6,7,8,9$ & 3 & 2 & 0 & 0 & 0.9 & 0.015 \\
\hline$\{4,9\}$ & $1,3,7,8$ & 4 & 0 & 0 & 0.5 & 0 & 0.0144 \\
\hline$\{4,10\}$ & $1,3,8$ & 2 & 0 & 1 & 0 & 0.7 & 0.042 \\
\hline$\{5,10\}$ & $1,2,6,8,9$ & 2 & 1 & 2 & 0 & 0.8 & 0.067 \\
\hline$\{6,9\}$ & $1,3,5,8,10$ & 3 & 1 & 2 & 0 & 0.8 & 0.067 \\
\hline$\{7,8\}$ & $3,4,5,6,9$ & 5 & 0 & 0 & 0 & 0.8 & 0.426 \\
\hline$\{7,10\}$ & $1,3,6,9$ & 3 & 0 & 1 & 0 & 0.8 & 0.188 \\
\hline
\end{tabular}

In the Table 1, we have shown the relation between the users as per the notions stated above. The relationship between the two users is considered as symmetric and bidirectional which means if there is a link between $\mathrm{U} 1$ and $\mathrm{U} 2$, there must also a link between $\mathrm{U} 2$ and $\mathrm{U} 1$.

Now let's think of triadic closure. If we can have an in-depth view over Table 1, we can see that there are number of possibilities by which triadic closures can exist following the baseline model as shown in Table. 2 above.

Table-3 Relation Matrix of the Observation in August, 2016

\begin{tabular}{|c|c|c|c|c|c|c|c|c|c|c|}
\hline & $\mathrm{U} 1$ & $\mathrm{U} 2$ & U3 & U4 & U5 & U6 & U7 & U8 & U9 & $\begin{array}{c}\mathrm{U} 1 \\
0\end{array}$ \\
\hline U1 & - & 1 & 0 & 1 & 1 & 1 & 1 & 0 & 1 & 1 \\
\hline U2 & 1 & - & 1 & 0 & 1 & 1 & 0 & 1 & 0 & 1 \\
\hline $\mathrm{U} 3$ & 0 & 1 & - & 1 & 0 & 1 & 1 & 1 & 1 & 1 \\
\hline $\mathrm{U} 4$ & 1 & 0 & 1 & - & 0 & 0 & 1 & 1 & 0 & 1 \\
\hline U5 & 1 & 1 & 0 & 0 & - & 1 & 1 & 1 & 1 & 0 \\
\hline U6 & 1 & 1 & 1 & 0 & 1 & - & 1 & 1 & 0 & 1 \\
\hline U7 & 1 & 0 & 1 & 1 & 1 & 1 & - & 1 & 1 & 1 \\
\hline U8 & 0 & 1 & 1 & 1 & 1 & 1 & 1 & - & 1 & 1 \\
\hline U9 & 1 & 0 & 1 & 0 & 1 & 0 & 1 & 1 & - & 1 \\
\hline $\begin{array}{c}\mathrm{U} 1 \\
0\end{array}$ & 1 & 1 & 1 & 1 & 0 & 1 & 1 & 1 & 1 & - \\
\hline
\end{tabular}


The cell values in the Table- 3 depicts whether user are in friendship relation or not. If the cell values are 1 it means, they are connected. The highlighted values signify to the fact that they have created the new triadic closure. The graphical view of the observation is shown in Figure-2 below where the darken lines have been emerged due to social influence along with inclination to a particular context.

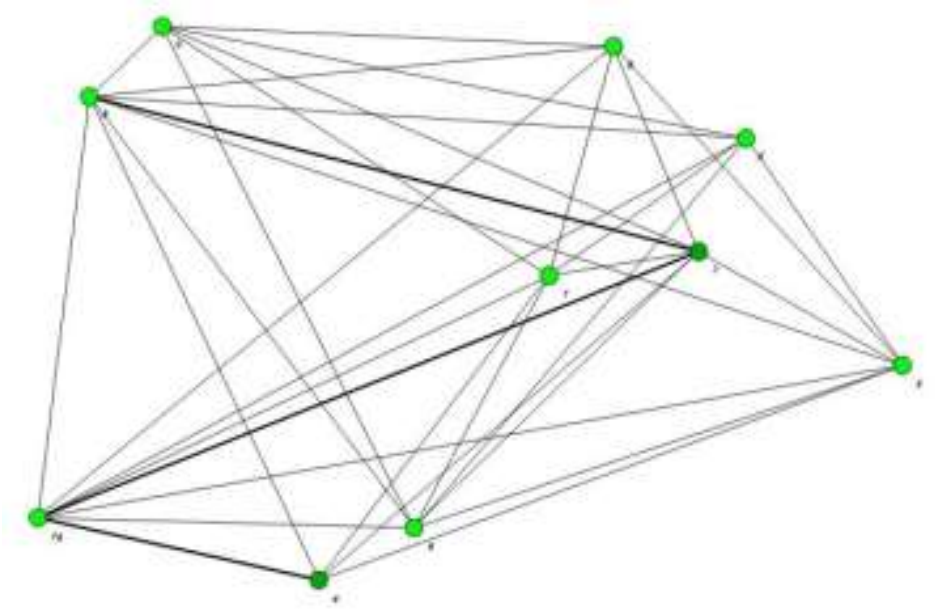

Figure 2. Connection Graph Observed in August 20, 2016

If we observe the values carefully in the Table-2, it shows a significant effect of inclination of users on a particular context. If, a user has no mind to be in a context even though there are friends or mutual friends in that context, there will be no new link generation and therefore no triadic closure can emerge. This can be explained as:

$$
\begin{gathered}
\text { if, } p_{\text {inc }}=0 \text { then, } \varrho^{k} \times p_{\text {inc }}=0, \text { as a result, } \\
1-(1-p) e^{k} \times p_{\text {inc }}=1-(1-p)^{0}=1-1=0
\end{gathered}
$$

Therefore, we can say that $\varrho^{k} \times p_{i n c}$ has its significant contribution in this proposed probability model. If we look at the contribution, we can easily understand that the changes of relationship occur only when the two users possess the same influence exposure. Therefore, the contribution of contextual influence with number of mutual friends is highly significant for the social networking relations and its analysis.

We have also compared our proposed probabilistic model with the generic triadic model with respect to convergence to a stable network in social network paradigm and erroneous detection of probable mutual friends. Table -4 and Table-5 represent the comparison.

Table 4. Error Percentage Comparison

\begin{tabular}{|c|c|c|}
\hline & \multicolumn{2}{|c|}{ Error percentage in probability of triads } \\
\hline No. of users & Non Probabilistic Model & Proposed Probabilistic Model \\
\hline 10 & 11.39 & 2.11 \\
\hline 20 & 12.44 & 2.33 \\
\hline 30 & 12.666 & 3.33 \\
\hline 40 & 13.933 & 3.67 \\
\hline 50 & 14.001 & 3.877 \\
\hline
\end{tabular}

The above data represent that the probabilistic model giver a better comprehension for understanding the triads as the error in calculating the mutual friends is less in our 
proposed model. We have also compared our proposed model with respect to convergence to stable network. The convergence comparison results as shown below in Table-5 emphasizes that the existing non probabilistic model is faster in converging to a stable network or close knit group which is not actual fact in the social networks. Our proposed model converges with a larger number of mutual friends as compared to the existing nonprobabilistic model.

Table 5. Convergence Comparison with Mutual Friends

\begin{tabular}{|c|c|c|}
\hline \multirow{2}{*}{} & \multicolumn{2}{|c|}{ Convergence with mutual friends } \\
\cline { 2 - 3 } & $\begin{array}{l}\text { Non Probabilistic Model } \\
\text { (no. of probable mutual friends) }\end{array}$ & $\begin{array}{l}\text { Proposed Probabilistic Model } \\
\text { (no. of probable mutual friends) }\end{array}$ \\
\hline After 1 month & 12 & 4 \\
\hline After 2 month & 32 & 17 \\
\hline After 3 month & 47 & 21 \\
\hline
\end{tabular}

The data in Table 5 shows that the proposed model detects less number of probable mutual friends as compared to the non-probabilistic model. But the emphasized fact is our proposed model identifies the probable mutual friends with better accuracy. As a result, the proposed model creates a strong base for analysing the strongly knit group and their related dynamic behaviour.

\section{Conclusion}

Our study of the facts and figures and the above analysis thus proves that our proposed theory of Probabilistic Triadic Model, measuring triadic closure in absolute way for the real working scenarios as compared to the existing theory. Besides, we have also seen how our proposed theory can provide the exact interpretation of the facts. It can be applicable with any kind of social structure to check the effect social influences of social bonds. This analysis has been studied by us on triadic closure till now. Our proposed model will be more beneficial to analyse the social networking aspects and the social links in different social networking websites and also the presence of social influences. In our future work, we shall try this probabilistic triadic model following the strong and weak ties with bridges and local bridges formation. Further this probabilistic model will also help to analyse the social data more precisely.

\section{References}

[1] T.-Q. Peng,, "Assortative mixing, preferential attachment, and triadic closure. A longitudinal study of tie-generative mechanisms in journal citation networks", Journal of Informetrics, vol. 9, no. 2, (2015), pp. 250-262.

[2] T. Lou, J. Tang, J. Hopcroft, Z. Fang and X. Ding, "Learning to predict reciprocity and triadic closure in social networks", ACM Transactions on Knowledge Discovery from Data (TKDD), vol. 7, no. 2, (2013), pp. 5.1-5.25.

[3] P. Klimek and S. Thurner, "Triadic closure dynamics drives scaling-laws in social multiplex networks", New Journal of Physics, vol. 15, no. 6, (2013).

[4] M. Li, H. Zou, S. Guan, X. Gong, K. Li, Z. Di and C.-H. Lai, "A coevolving model based on preferential triadic closure for social media networks", Scientific Reports, vol. 3, Nature Science, (2013).

[5] M. Zignani, S. Gaito, G. P. Rossi, X. Zhao, H. Zheng and B. Y. Zhao, "Link and Triadic Closure Delay: Temporal Metrics for Social Network Dynamics", Proceedings of the Eighth International AAAI Conference on Weblogs and Social Media, (2014); Michigan, USA. 
[6] P. Grindrod, D. J. Higham and M. C. Parsons, "Bistability through Triadic Closure", Internet Mathematics, vol. 8, no. 4, (2012), pp. 402-423.

[7] A. L. J. Ter Wal., "The dynamics of the inventor network in German biotechnology: geographic proximity versus triadic closure", Journal of Economic Geography, vol. 14, no. 3, (2014), pp. 589-620.

[8] C. Seshadhri, Ali Pinar, Nurcan Durak and Tamara G. Kolda, "Directed closure measures for networks with reciprocity", Journal of Complex Networks, (2016).

[9] H. Huang, J. Tang, S. Wu, L. Liu and X. Fu, "Mining Triadic Closure Patterns in Social Networks", International World Wide Web Conference Committee (IW3C2), Seoul, Korea, (2014), April 7-11.

[10] G. Kossinets and D. Watts, "Empirical analysis of an evolving social network", Science Magazine, vol.311, no. 5757, (2006), pp. 88-90.

[11] D. Easley and J. Kleinberg, "Networks, Crowds, and Markets: Reasoning about a Highly Connected World", Cambridge University Press, (2010), pp. 98-102.
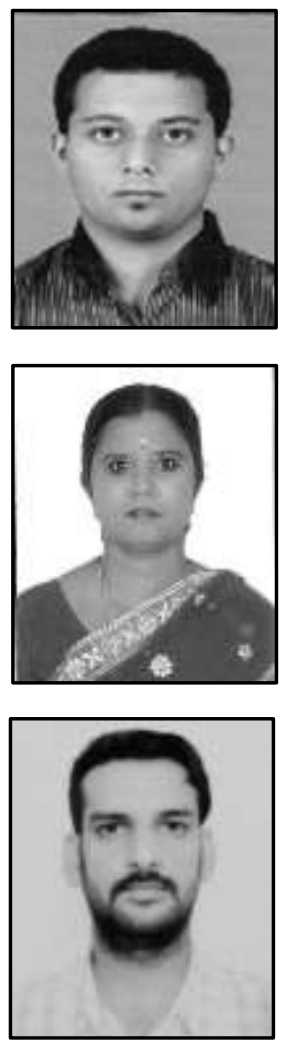

\section{Authors}

Rahul Saha is working as Assistant Professor in Lovely Professional University, Punjab, India and pursuing Ph.D in Computer Engineering from the same. His research interests include cryptography and network security, information security, wireless sensor networks and social networking analysis. He has published more than twenty research papers in various international refereed journals and conferences.

G. Geetha is the Dean of Research and Development in Lovely Professional University, Punjab, India. Her research interest includes Cryptography and Software Engineering. She has published more than 50 papers in refereed Journals and Conferences. She is also the Editorial Board of IJACM and IJCRYPTO. She is presently the President of Advanced Computing Research Society.

Gulshan Kumar working as an Assistant Professor in Lovely Professional University, Punjab India and did his B.Tech from Amritsar College of Engineering, Amritsar (2009) in Computer Science Engineering, M.Tech from Lovely Professional University, Punjab, India with area of specialization in Mobile Ad hoc and Sensor Networks. He has many publications in well renowned International journals and Conferences. 